\title{
On the generalized Davenport constant and the Noether number
}

\author{
Kálmán Cziszter ${ }^{a *}$ and $\quad$ Mátyás Domokos ${ }^{b \dagger}$ \\ ${ }^{a}$ Central European University, Department of Mathematics and its Applications, \\ Nádor u. 9, 1051 Budapest, Hungary \\ Email: cziszter_kalman-sandor@ceu-budapest.edu \\ ${ }^{b}$ Rényi Institute of Mathematics, Hungarian Academy of Sciences, \\ Reáltanoda u. 13-15, 1053 Budapest, Hungary \\ Email: domokos.matyas@renyi.mta.hu
}

\begin{abstract}
Known results on the generalized Davenport constant relating zerosum sequences over a finite abelian group are extended for the generalized Noether number relating rings of polynomial invariants of an arbitrary finite group. An improved general upper degree bound for polynomial invariants of a non-cyclic finite group that cut out the zero vector is given. 2010 MSC: 13A50 (Primary) 11B75, $13 \mathrm{~A} 02$ (Secondary)

Keywords: Noether number, Davenport constant, polynomial invariants
\end{abstract}

\section{Introduction}

The Davenport constant $\mathrm{D}(A)$ of a finite abelian group $A$ is defined as the smallest positive integer $n$ such that every sequence over $A$ of length at least $n$ has a non-empty zero-sum subsequence. The Davenport constant naturally appears in the theory of polynomial invariants of finite groups. The Noether number $\beta(G)$ of a finite group $G$ is the maximal possible degree of an indecomposable polynomial invariant of $G$ (the definition involves a fixed base field $\mathbb{F}$ which we always assume to have characteristic not dividing the order of $G$ ). It was shown by B. Schmid [20] that when $G=A$ is abelian, then $\beta(G)=\mathrm{D}(A)$.

Halter-Koch [14 introduced for any positive integer $k$ the generalized Davenport constant $\mathrm{D}_{k}(A)$ as the smallest positive integer $n$ such that every sequence over $A$ of length at least $n$ is divisible by the product of $k$ non-empty zero-sum subsequences (cf. Chapter 6.1 in [13]; by the product of sequences over $A$ we mean their concatenation, and divisibility of sequences is defined accordingly).

\footnotetext{
${ }^{*}$ The paper is based on results from the $\mathrm{PhD}$ thesis of the first author written at the Central European University.

${ }^{\dagger}$ The second author is partially supported by OTKA NK81203 and K101515.
} 
This notion also can be seen as the abelian special case of a concept of invariant theory. In [2] the authors introduced the generalized Noether number $\beta_{k}(G)$ for an arbitrary finite group $G$ and positive integer $k$ (the definition again involves a fixed base field $\mathbb{F}$ of characteristic not dividing $|G|$, suppressed from the notation). When $G=A$ is abelian, then $\beta_{k}(A)=\mathrm{D}_{k}(A)$. As it is demonstrated in [2], the main use of the generalized Noether number is that it can be efficiently used to derive upper bounds for the ordinary Noether number of a group in terms of the generalized Noether numbers of its subquotients (see the beginning of Section 4 for some details).

In the first part of the present note we develop further the analogy between the generalized Davenport constant and the generalized Noether number. Theorem 3.4 gives a lower bound for the generalized Noether number of a direct product of groups in terms of the generalized Noether numbers of the factors, generalizing thereby Lemma 6.1.4 from [13 for non-abelian groups. Next we investigate the behaviour of $\beta_{k}(G)$ as a function of $k$. The fact that $D_{k}(A)$ is an almost linear function of $k$ was shown by M. Freeze and W. A. Schmid 11, building on a result of Delorme, Ordaz and Quiroz [4]; see also Theorem 6.1.5 in [13. This is generalized in Corollary 4.7, which we derive from basic generalities on graded rings and the method of polarization in invariant theory. It states that for a fixed finite group $G$ and a base field $\mathbb{F}$ of characteristic zero there exists a positive integer $k_{0}$ and a non-negative integer $\beta_{0}(G)$ such that for all $k \geq k_{0}$ we have $\beta_{k}(G)=k \sigma(G)+\beta_{0}(G)$; the number $\sigma(G)$ is another well known quantity of invariant theory: it is the minimal positive integer $n$ such that for any representation of $G$ there exist homogeneous $G$-invariant polynomial functions of degree at most $n$ such that their only common zero is the origin. (In the special case when $G=A$ is abelian, we have $\sigma(A)=\exp (A)$, the exponent of $A$. We note also that for linearly reductive algebraic groups a related quantity $\sigma(G, V)$ plays a significant role in constructive invariant theory, see [19] or [5].)

In Section 5 we establish some basic properties of $\sigma(G)$ for $G$ finite. They are used to prove Theorem 7.1. stating that if $G$ is non-cylic, then $\sigma(G) \leq|G| / q$ for the minimal prime divisor of $|G|$, provided that char $(\mathbb{F})$ does not divide $|G|$. This is an easier but stronger variant for $\sigma(G)$ of the main combined result on $\beta(G)$ proved in [2] and [3] (continuing the investigations of [20, [7, 21]).

\section{Preliminaries}

We need to recall some generalities on graded modules. By a graded module here we mean an $\mathbb{N}$-graded module $M=\bigoplus_{d=0}^{\infty} M_{d}$ over a commutative graded $\mathbb{F}$ algebra $R=\bigoplus_{d=0}^{\infty} R_{d}$ such that $R_{0}=\mathbb{F}$ is a field when $R$ is unital and $R_{0}=\{0\}$ otherwise; in the latter case still we assume that $M$ is an $\mathbb{F}$-vector space, the multiplication map is $\mathbb{F}$-bilinear, and a submodule or an ideal by definition is assumed to be a subspace. We set $M_{\leq s}:=\bigoplus_{d=0}^{s} M_{d}$, and $R_{+}:=\bigoplus_{d>0} R_{d}$. We write $R_{+}^{l}$ for the $l$ th power of the ideal $R_{+}$, and more generally, for subsets $A, B \subset R, A B$ stands for the $F$-subspace spanned by $\{a b \mid a \in A, b \in B\}$, and $\langle A\rangle$ denotes the ideal in $R$ generated by $A$. The subalgebra of $R$ generated by 
$R_{\leq s}:=\bigoplus_{d=0}^{s} R_{d}$ will be denoted by $\mathbb{F}\left[R_{\leq s}\right]$.

The factor space $M / R_{+} M$ inherits the grading. Define

$$
\beta(M, R):=\text { the top degree of } M / R_{+} M
$$

provided that $M / R_{+} M$ has finitely many non-zero homogeneous components, and write $\beta(M, R)=\infty$ otherwise. By the graded Nakayama Lemma $\beta(M, R)$ is the minimal non-negative integer $s$ such that $M=M_{\leq s}+M_{\leq s} R$ (i.e. $M$ is generated as an $R$-module by $M_{\leq s}$ ), when $M$ is generated in bounded degree.

In particular, the maximal degree of a homogeneous element of $R_{+}$not belonging to $R_{+}^{2}$ is

$$
\beta(R):=\beta\left(R_{+}, R\right),
$$

the minimal positive integer $n$ such that $R$ is generated as an $\mathbb{F}$-algebra by homogeneous elements of degree at most $n$.

Let $M$ be a graded $R$-module. We define for any integer $k \geq 1$

$$
\beta_{k}(M, R):=\beta\left(M, R_{+}^{k}\right)
$$

The abbreviation

$$
\beta_{k}(R):=\beta_{k}\left(R_{+}, R\right)
$$

will also be used. In the special case $k=1$ we recover $\beta_{1}(M, R)=\beta(M, R)$ and $\beta_{1}(R)=\beta(R)$. Note the trivial inequality $\beta_{k}(R) \leq k \beta(R)$.

The graded modules and algebras we are interested in come from invariant theory. We fix a base field $\mathbb{F}$, and let $G$ be a finite group whose order is invertible in $\mathbb{F}$. Take a $G$-module $V$, i.e. a finite dimensional $\mathbb{F}$-vector space endowed with a representation of $G$ on $V$. The coordinate ring $\mathbb{F}[V]$ is defined in abstract terms as the symmetric tensor algebra of the dual space $V^{*}$. So $\mathbb{F}[V]$ is a polynomial ring in $\operatorname{dim}(V)$ variables, hence in particular it is a graded ring with $\mathbb{F}[V]_{1}=V^{*}$. The left action of $G$ on $V$ induces a natural right action on $V^{*}$ given by $x^{g}(v)=x(g v)$ for any $g \in G, v \in V$ and $x \in V^{*}$. This right action of $G$ on $V^{*}$ extends to the symmetric tensor algebra $\mathbb{F}[V]$. The corresponding ring of polynomial invariants is

$$
\mathbb{F}[V]^{G}:=\left\{f \in \mathbb{F}[V]: f^{g}=f \quad \forall g \in G\right\}
$$

and

$$
\beta(G, V):=\beta\left(\mathbb{F}[V]^{G}\right)
$$

is called the Noether number of the $G$-module $V$. We also set

$$
\beta_{k}(G, V):=\beta_{k}\left(\mathbb{F}[V]^{G}\right)
$$

for an arbitrary positive integer $k$, and

$$
\beta_{k}(G):=\sup \left\{\beta_{k}(G, V) \mid V \text { is a finite dimensional } G \text {-module over } \mathbb{F}\right\} .
$$

We shall refer to these numbers as the generalized Noether numbers of the group $G$. 
The famous theorem of $\mathrm{E}$. Noether asserts that $\beta(G):=\beta_{1}(G)$ is bounded by the order of $G$. When $\operatorname{char}(\mathbb{F})=0$ or $\operatorname{char}(\mathbb{F})>|G|$, this was proved in [18. The result was extended to non-modular positive characteristic independently by Fleischmann [8] and Fogarty [10].

Convention 2.1. Throughout this paper $\mathbb{F}$ is our base field and $G$ (or $H$ ) is a finite group of order not divisible by $\operatorname{char}(\mathbb{F})$, unless explicitly stated otherwise. By a $G$-module we mean a finite dimensional $\mathbb{F}$-vector space endowed with a linear action of $G$.

\section{Lower bound for direct products}

Lemma 3.1. For any $G$-module $V$ there exists an irreducible $G$-module $U$ such that

$$
\beta_{k}(G, V \oplus U) \geq \beta_{k}\left(\mathbb{F}[V], \mathbb{F}[V]^{G}\right)+1 .
$$

Proof. Write $L:=\mathbb{F}[V], R:=\mathbb{F}[V]^{G}$ and set $d:=\beta_{k}(L, R)$. By complete reducibility of the $G$-module $L_{d}$ its submodule $R_{+}^{k} L \cap L_{d}$ has a direct complement, which is non-zero by the definition of $d$, hence it contains an irreducible $G$-submodule $U$. Choose a basis $e_{1}, \ldots, e_{n}$ in $U$ and let $\varepsilon_{1}, \ldots, \varepsilon_{n}$ be the corresponding dual basis in $U^{*}$. The matrix of $g$ acting on $U^{*}$ via the contragredient representation is the transpose of the inverse of the matrix of $g$ acting on $U$. Thus $f:=\sum_{i=1}^{n} e_{i} \varepsilon_{i}$, viewed as an element in the polynomial ring $\mathbb{F}[V \oplus U]=\mathbb{F}[V] \otimes \mathbb{F}\left[\varepsilon_{1}, \ldots, \varepsilon_{n}\right]$, is a $G$-invariant of degree $d+1$. We claim that $f \notin S_{+}^{k+1}$ where $S:=\mathbb{F}[V \oplus U]^{G}$. Note that the action of $G$ on $\mathbb{F}[V \oplus U]$ preserves the total degree both in the variables belonging to $V^{*}$ and to $U^{*}$. Suppose indirectly that $f \in S_{+}^{k+1}$. Then $f=\sum_{j} g_{j} h_{j}$ where $g_{j} \in R_{+}^{k}$ while $h_{j} \in S_{+}$is linear on $U$, i.e. $h_{j}=\sum_{i=1}^{n} h_{j, i} \varepsilon_{i}$ for some polynomials $h_{j, i} \in L$. After equating the coefficients of $\varepsilon_{i}$ on both sides we get that $e_{i}=\sum_{j} g_{j} h_{j, i} \in R_{+}^{k} L$, contradicting the choice of $U$.

Corollary 3.2. If $V$ is a $G$-module such that $\beta_{k}(G, V)=\beta_{k}(G)$ then

$$
\beta_{k}(G, V)=\beta_{k}\left(\mathbb{F}[V], \mathbb{F}[V]^{G}\right)+1 .
$$

Proof. For any $G$-module $V$ it holds that $\beta_{k}(G, V) \leq \beta_{k}(L, R)+1$ where $L=$ $\mathbb{F}[V]$ and $R=\mathbb{F}[V]^{G}$. Indeed, if $f \in L$ has degree $\operatorname{deg}(f)>\beta_{k}(L, R)+1$ then $f \in L_{+} \cdot\left(\bigoplus_{d>\beta_{k}(L, R)} L_{d}\right) \subseteq L_{+} R_{+}^{k}$. Apply the transfer map $\tau: L \rightarrow R$, $f \mapsto \sum_{g \in G} f^{g}$. It is a graded $R$-module epimorphism from $L$ onto $R$ (see for example Chapter 1 in [1]). It follows that $\tau(f) \in R_{+}^{k+1}$, and on the other hand $\tau(L)=R$, hence the desired inequality follows. The reverse inequality is an immediate consequence of Lemma 3.1 .

Remark 3.3. Compare Corollary 3.2 with the formula $\mathrm{D}_{k}(A)=\mathrm{d}_{k}(A)+1$ in Lemma 6.1 .2 of [13], where $\mathrm{d}_{k}(A)$ is the maximal length of a sequence over $A$ which is not divisible by the product of $k$ zero-sum subsequences. It is not difficult to show that $\mathrm{d}_{k}(A)=\sup _{V} \beta_{k}\left(\mathbb{F}[V], \mathbb{F}[V]^{A}\right)$ where $V$ ranges over all $A$-modules, whereas $\beta_{k}(A)=\mathrm{D}_{k}(A)$, as we mentioned in the introduction. 
Theorem 3.4. For any integers $r, s \geq 1$ and finite groups $G, H$ we have the inequality

$$
\beta_{r+s-1}(G \times H) \geq \beta_{r}(G)+\beta_{s}(H)-1 .
$$

Proof. If $M$ and $N$ are graded modules over the graded algebras $R$ and $S$, respectively, then:

$$
\beta_{r+s-1}(M \otimes N, R \otimes S) \geq \beta_{r}(M, R)+\beta_{s}(N, S)
$$

Indeed, there are elements $x \in M_{\beta_{r}(M, R)} \backslash R_{+}^{r} M$ and $y \in N_{\beta_{s}(N, S)} \backslash S_{+}^{s} N$. Take an $\mathbb{F}$-vector space basis $\mathcal{B}_{1}$ of $R_{+}^{r} M$, and extend $\mathcal{B}_{1} \cup\{x\}$ to a basis $\mathcal{B}$ of $M$. Similarly, let $\mathcal{C}_{1}$ be a basis of $S_{+}^{s} N$, and extend $\mathcal{C}_{1} \cup\{y\}$ to a basis $\mathcal{C}$ in $N$. Then $\mathcal{A}:=\left\{u \otimes v \mid u \in \mathcal{B}_{1}, v \in \mathcal{C}\right.$ or $\left.u \in \mathcal{B}, v \in \mathcal{C}_{1}\right\}$ is a basis of $T:=R_{+}^{r} M \otimes N+M \otimes S_{+}^{s} N$. On the other hand $\mathcal{A} \cup\{x \otimes y\}$ is part of the basis $\{u \otimes v \mid u \in \mathcal{B}, v \in \mathcal{C}\}$ of $M \otimes N$, showing that $x \otimes y \notin T$. But $T \supseteq(R \otimes S)_{+}^{r+s-1}(M \otimes N)$ and $\operatorname{deg}(x \otimes y)=\beta_{r}(M, R)+\beta_{s}(N, S)$, whence (11) readily follows.

Now take a $G$-module $V$ with $\beta_{r}(G, V)=\beta_{r}(G)$, and an $H$-module $W$ with $\beta_{s}(H, W)=\beta_{s}(H)$. Given that $\mathbb{F}[V \oplus W]^{G \times H}=\mathbb{F}[V]^{G} \otimes \mathbb{F}[W]^{H}$ we have the following sequence of inequalities:

$$
\begin{aligned}
\beta_{r+s-1}(G \times H)-1 & \geq \beta_{r+s-1}\left(\mathbb{F}[V \oplus W], \mathbb{F}[V \oplus W]^{G \times H}\right) & & \text { by Lemma 3.1 } \\
& \geq \beta_{r}\left(\mathbb{F}[V], \mathbb{F}[V]^{G}\right)+\beta_{s}\left(\mathbb{F}[W], \mathbb{F}[W]^{H}\right) & & \text { by } 1 \text { (1) } \\
& =\beta_{r}(G)+\beta_{s}(H)-2 & & \text { by Corollary 3.2 }
\end{aligned}
$$

Remark 3.5. In the special case $G=A$ abelian we recover Lemma 6.1.4 from [13.

\section{The growth rate of $\beta_{k}(G)$}

In the study of the Noether bound for finite groups, the following inequalities due to Schmid 20] in characteristic zero and extended to positive non-modular characteristic by Sezer 21, Fleischmann [9, Knop [16] are very useful: For a normal subgroup $N$ in $G$ and an arbitrary subgroup $H$ in $G$ we have $\beta(G) \leq$ $[G: H] \beta(H)$ and $\beta(G) \leq \beta(N) \beta(G / N)$. Our motivation to study $\beta_{k}(G)$ stems from the following strengthening proved in [2]:

$$
\begin{aligned}
& \beta_{k}(G) \leq \beta_{\beta_{k}(G / N)}(N) \\
& \beta_{k}(G) \leq \beta_{k[G: H]}(H)
\end{aligned}
$$

The estimates for $\beta(G)$ obtained in terms of generalized Noether numbers of its subquotient $K$ using the latter inequalities are better than the estimates derived from the original inequalitites, as soon as $\beta_{k}(K)$ is strictly smaller than $k \beta(K)$. A partial theoretical explanation of the experience that indeed, typically $\beta_{k}(G)$ is strictly smaller than $k \beta(G)$ and its extent is obtained in this section. 
We start by studying in general for a fixed commutative graded $\mathbb{F}$-algebra $R$ the behavior of $\beta_{k}(R)$ as a function of $k$. The surjection $R_{+} / R_{+}^{k+1} \rightarrow R_{+} / R_{+}^{k}$ shows that $\beta_{k}(R) \leq \beta_{k+1}(R)$ for all $k$. We note that $\beta_{k}(R)$ is not always a strictly increasing function of $k$ :

Example 4.1. Consider the ring $R=\mathbb{F}[a, b] /\left(b^{3}-a^{9}, a b^{2}-a^{7}\right)$ and define a grading by setting $\operatorname{deg}(a)=1$ and $\operatorname{deg}(b)=3$. Then $b^{2} \in R_{+}^{2} \backslash R_{+}^{3}$, and $b^{2}$ spans the degree 6 homogeneous component of $R_{+}^{2} / R_{+}^{4}$. In this case for all $l \geq 7$ we have that $R_{l} \subseteq R_{+}^{5}$, hence $6=\beta_{2}(R)=\beta_{3}(R)=\beta_{4}(R)$.

On the other hand, for a fixed $G$-module $V, \beta_{k}(G, V)$ is unbounded because of the following trivial observation:

Lemma 4.2. $\beta_{k}(R)$ as a function of $k$ is bounded if and only if there is an integer $n$ such that $R_{i}=\{0\}$ for all $i \geq n$.

Proof. Note that $R_{+}^{n+1} \subseteq \bigoplus_{d>n+1} R_{d}$. Hence if $R_{n} \neq\{0\}$, then $R_{n} \nsubseteq R_{+}^{n+1}$, implying $\beta_{n}(R) \geq n$. Conversely, if $R_{i}=\{0\}$ for all $i \geq n$ then $\beta_{i}(R)<n$.

Lemma 4.3. For any positive integers $r \leq k$ we have the inequality

$$
\beta_{k}(R) \leq \frac{k}{r} \beta_{r}(R)
$$

Proof. Suppose to the contrary that $\beta_{k}(R)>\frac{k}{r} \beta_{r}(R)$. Then there exist homogeneous elements $f_{1}, \ldots, f_{l} \in R_{+}$such that $l \leq k, f:=f_{1} \cdots f_{l}$ is not contained in $R_{+}^{l+1}$, and $\operatorname{deg}(f)>\frac{k}{r} \beta_{r}(R)$ (this forces that $l>r$ ). We may suppose that $\operatorname{deg}\left(f_{1}\right) \geq \cdots \geq \operatorname{deg}\left(f_{l}\right)$. Then

$$
\frac{\operatorname{deg}\left(f_{1}\right)+\cdots+\operatorname{deg}\left(f_{r}\right)}{r} \geq \operatorname{deg}\left(f_{r}\right) \geq \operatorname{deg}\left(f_{r+1}\right) \geq \frac{\operatorname{deg}\left(f_{r+1}\right)+\cdots+\operatorname{deg}\left(f_{l}\right)}{l-r}
$$

hence

$$
\operatorname{deg}\left(f_{1} \ldots f_{r}\right) \geq \frac{r}{l} \operatorname{deg}\left(f_{1} \ldots f_{l}\right) \geq \frac{r}{k} \operatorname{deg}(f)>\beta_{r}(R) .
$$

It follows that $h:=f_{1} \cdots f_{r} \in R_{+}^{r+1}$, hence $f=h f_{r+1} \cdots f_{l} \in R_{+}^{l+1}$, a contradiction.

By Lemma 4.3 the sequence $\frac{\beta_{k}(R)}{k}$ is monotonically decreasing, and as it is also non-negative, it must converge to a certain limit. Our next goal will be to clarify what is the value of this limit. For a graded finitely generated commutative $\mathbb{F}$-algebra $R$ with $R_{0}=\mathbb{F}$ set

$$
\sigma(R):=\min \left\{d \in \mathbb{N}: R \text { is finitely generated as a module over } \mathbb{F}\left[R_{\leq d}\right]\right\}
$$

Equivalently, $\sigma(R)$ is the minimal integer $d$ such that $\beta\left(R_{+}, \mathbb{F}\left[R_{\leq}\right]\right)$is finite.

Proposition 4.4. Let $R$ be a finitely generated commutative graded $\mathbb{F}$-algebra. For any positive integer $k$ we have $\beta_{k}(R) \geq k \sigma(R)$. 
Proof. It is well known that given a set $h_{1}, \ldots, h_{s} \in R$ of homogeneous elements, $R$ is a finitely generated module over its subalgebra $\mathbb{F}\left[h_{1}, \ldots, h_{s}\right]$ if and only if $R_{+}=\sqrt{\left\langle h_{1}, \ldots, h_{s}\right\rangle}$, the radical of the ideal generated by the $h_{i}$. If $R$ is finite dimensional then $\sigma(R)=0$ and our statement obviously holds. Suppose $\operatorname{dim}_{\mathbb{F}}(R)=\infty$, hence $\sigma(R)>0$. By definition of $t:=\sigma(R)$ we have $R_{+} \neq$ $\sqrt{\left\langle\bigoplus_{d=1}^{t-1} R_{d}\right\rangle}$, on the other hand $R_{+}=\sqrt{\left\langle\bigoplus_{d=1}^{t} R_{d}\right\rangle}$, hence there exists an $f \in R_{t}$ with $f \notin \sqrt{\left\langle\bigoplus_{d=1}^{t-1} R_{d}\right\rangle}$. We claim that $f^{k} \notin R_{+}^{k+1}$. Indeed, $f^{k} \in R_{+}^{k+1}$ would imply $f^{k} \in\left\langle\bigoplus_{d=1}^{t-1} R_{d}\right\rangle$, contrary to the choice of $f$. Thus $\beta_{k}(R) \geq$ $\operatorname{deg}\left(f^{k}\right)=k \sigma(R)$.

By definition of $\sigma(R)$ the number

$$
\eta(R):=\beta\left(R_{+}, \mathbb{F}\left[R_{\leq \sigma(R)}\right]\right)
$$

is finite. Moreover, any homogeneous element $f \in R$ with $\operatorname{deg}(f)>\eta(R)$ belongs to the ideal $\left(\bigoplus_{d=1}^{\sigma(R)} R_{d}\right) \cdot R_{+}$, hence $\beta(R) \leq \eta(R)$ and more generally, by induction on $k$ one obtains

$$
\beta_{k}(R) \leq(k-1) \sigma(R)+\eta(R) .
$$

We know from Lemma 4.2 that an integer $k_{0}$ exists such that $\beta_{k}(R) \geq \eta(R)-$ $\sigma(R)$ holds for any $k \geq k_{0}$. Hence if $\operatorname{deg}(f)>\beta_{k}(R)+\sigma(R)$ then $f \in R$ can be written in the form $\sum_{i} g_{i} h_{i}$ where $0<\operatorname{deg}\left(g_{i}\right) \leq \sigma(R)$ and $\operatorname{deg}\left(h_{i}\right)>\beta_{k}(R)$, whence $h_{i} \in R_{+}^{k+1}$ and $f \in R_{+}^{k+2}$. This argument shows that for any $k \geq k_{0}$ we have

$$
\beta_{k+1}(R) \leq \beta_{k}(R)+\sigma(R)
$$

This simple observation immediately leads us to the following result:

Proposition 4.5. Let $R$ be a finitely generated commutative graded $\mathbb{F}$-algebra. Then there are non-negative integers $k_{0}(R)$ and $\beta_{0}(R)$ such that

$$
\beta_{k}(R)=k \sigma(R)+\beta_{0}(R) \quad \text { for every } k>k_{0}(R) .
$$

Proof. Consider the sequence of integers $a_{k}:=\beta_{k}(R)-k \sigma(R)$, where $k=$ $k_{0}, k_{0}+1, \ldots$ and $\beta_{k_{0}}(R) \geq \eta(R)-\sigma(R)$. By (2) it is monotonically decreasing and by Proposition 4.4 it is non-negative, therefore it stabilizes after finitely many steps, and this is what has been claimed.

For any $G$-module $V$ we write

$$
\sigma(G, V):=\sigma\left(\mathbb{F}[V]^{G}\right) .
$$

This quantity was much studied for $G$ a linearly reductive group (see e.g. [5] or [19]) and has the following well-known interpretation by the Hilbert Nullstellensatz: 
Proposition 4.6. $\sigma(G, V)$ is the minimal positive integer $n$ such that there exists a subset of $\mathbb{F}[V]_{+}^{G}$ consisting of homogeneous elements with degree at most $n$, whose common zero locus in $\overline{\mathbb{F}} \otimes_{\mathbb{F}} V$ is $\{0\}$ (where $\overline{\mathbb{F}}$ stands for the algebraic closure of $\mathbb{F}$ ).

Supposing $|G| \in \mathbb{F}^{\times}$we have $\sigma(G, V) \leq \beta(G, V) \leq|G|$, and define

$$
\sigma(G):=\sup \{\sigma(G, V) \mid V \text { is a } G \text {-module }\} .
$$

(In fact the inequality $\sigma(G) \leq|G|$ holds in the modular case $\operatorname{char}(\mathbb{F})|| G \mid$ as well, see Remark 5.6 (ii).) As an immediate corollary of Proposition 4.5 we obtain that for any $G$-module $V$ there exist non-negative integers $k_{0}(G, V)$ and $\beta_{0}(G, V)$ such that for all $k \geq k_{0}(G, V)$ we have $\beta_{k}(G, V)=k \sigma(G, V)+\beta_{0}(G, V)$. In characteristic zero the following can be proved:

Corollary 4.7. Suppose $\operatorname{char}(\mathbb{F})=0$ and let $G$ be a finite group. There exist non-negative integers $k_{0}(G)$ and $\beta_{0}(G)$ such that for all $k \geq k_{0}(G)$ we have

$$
\beta_{k}(G)=k \sigma(G)+\beta_{0}(G) .
$$

In particular,

$$
\lim _{k \rightarrow \infty} \frac{\beta_{k}(G)}{k}=\sigma(G) .
$$

Proof. Denoting by $V_{\text {reg }}$ the regular representation of $G$, we have that $\beta_{k}(G)=$ $\beta_{k}\left(G, V_{\text {reg }}\right)$ holds for all $k$ by the same argument as in the proof of the special case $k=1$ in 20] based on Weyl's theorem on polarization (cf. [22]). Hence the statement holds by Proposition 4.5.

Remark 4.8. As we mentioned in the Introduction, Corollary 4.7 in the special case when $G=A$ is abelian is due to M. Freeze and W. A. Schmid [11, and Delorme, Ordaz and Quiroz [4. For some results on $\eta(A)$ see e.g. 13$]$ ch. 5.7.

\section{Some basic properties of $\sigma(G)$}

In this section we collect some basic statements about $\sigma(G)$ that we will need to prove Theorem 7.1

Lemma 5.1. Let $V_{1}, \ldots, V_{n}$ be any $G$-modules and $W=V_{1} \oplus \cdots \oplus V_{n}$. Then

$$
\sigma(G, W)=\max _{i=1}^{n} \sigma\left(G, V_{i}\right)
$$

In particular $\sigma(G)=\max _{U} \sigma(G, U)$ where $U$ ranges over all isomorphism classes of irreducible G-modules.

Proof. Let $R=\mathbb{F}[W]^{G}$ and denote by $S_{i}$ the subalgebra of $\mathbb{F}\left[V_{i}\right]^{G}$ generated by its elements of degree at most $\sigma\left(G, V_{i}\right)$. As $\mathbb{F}[W]=\otimes_{i=1}^{n} \mathbb{F}\left[V_{i}\right]$ is obviously finitely generated as a $\otimes_{i=1}^{n} S_{i}$-module, and $\otimes_{i=1}^{n} S_{i} \subseteq \mathbb{F}\left[R_{\leq d}\right]$ where $d:=\max _{i=1}^{n} \sigma\left(G, V_{i}\right)$, it follows that $\sigma(G, W) \leq d$. 
For the reverse inequality let $T=\mathbb{F}\left[V_{i}\right]^{G}$ for a fixed $i$ and observe that the restriction to $V_{i}$ gives a graded algebra surjection $\psi: R \rightarrow T$. Hence the image under $\psi$ of a finite set of module generators of $R$ over its subalgebra $\mathbb{F}\left[R_{\leq \sigma(R)}\right]$ must generate $T=\psi(R)$ as a module over its subalgebra $\psi\left(\mathbb{F}\left[R_{\leq \sigma(R)}\right]\right)=\mathbb{F}\left[T_{\leq \sigma(R)}\right]$, as well. In particular $\sigma\left(G, V_{i}\right) \leq \sigma(G, W)$.

Remark 5.2. The number $\sigma\left(\mathbb{F}[W]^{G}\right)$ when $G$ is a linearly reductive group acting algebraically on $W$ plays important role in finding explicit upper bounds for $\beta\left(\mathbb{F}[W]^{G}\right)$, see [19] and [5]. Lemma $[5.1$ is special for finite groups, and does not hold in general for reductive algebraic groups, when it may well happen that $\mathbb{F}[V]^{G}=\mathbb{F}$, but $\mathbb{F}[V \oplus \cdots \oplus V]^{G}$ contains non-constant elements (for example, take as $V$ the natural module $\mathbb{F}^{n}$ over $G=S L_{n}(\mathbb{F})$ ).

For an abelian group $A$ denote by $\exp (A)$ the least common multiple of the orders of the elements of $A$.

Corollary 5.3. Let $A$ be an abelian group and suppose that $\mathbb{F}$ is algebraically closed of characteristic not dividing $|A|$. Then

$$
\sigma(A)=\exp (A)
$$

Proof. Lemma 5.1 asserts that $\sigma(A)=\max _{U} \sigma(A, U)$ where $U$ runs through the irreducible representations of $A$. These are all 1-dimensional, and if $U^{*}=\langle x\rangle$ then $\mathbb{F}[x]^{A}=\mathbb{F}\left[x^{e}\right]$ where $e \in \mathbb{N}$ is the order of the character char $i: A \rightarrow \mathbb{F}^{\times}$ defined by $x^{a}=\operatorname{chari}(a) x(a \in A)$. This readily implies our claim, as $A \cong \hat{A}$, where $\hat{A}:=\operatorname{hom}_{\mathbb{Z}}\left(A, \mathbb{F}^{\times}\right)$is the group of characters of $A$.

Lemma 5.4. Let $N$ be a normal subgroup of $G$ and $V$ a $G$-module. Then

$$
\sigma(G, V) \leq \sigma(G / N) \sigma(N, V) .
$$

Proof. Set $W:=\bigoplus_{d=1}^{\sigma(N, V)} \mathbb{F}[V]_{d}^{N}$ and denote by $S$ the subalgebra of $\mathbb{F}[V]^{N}$ generated by $W$. Then $S$ is a finite module over its finitely generated subalgebra $S^{G / N}=S^{G}$, and $\mathbb{F}[V]^{N}$ is a finite $S$-module, thus $\mathbb{F}[V]^{N}$ is a finite, hence noetherian $S^{G}$-module, implying in turn that its submodule $\mathbb{F}[V]^{G}$ is also a finite $S^{G}$-module. Write $\pi$ for the $\mathbb{F}$-algebra surjection $\mathbb{F}\left[W^{*}\right] \rightarrow S$ induced by the natural isomorphism between the linear component $\left(W^{*}\right)^{*}$ of the polynomial ring $\mathbb{F}\left[W^{*}\right]$ and $W \subset \mathbb{F}[V]^{N}$. By linear reductivity of $G / N, \pi$ maps $\mathbb{F}\left[W^{*}\right]^{G / N}$ onto $S^{G}$. Let $T$ be the $\mathbb{F}$-subalgebra of $\mathbb{F}\left[W^{*}\right]$ generated by $\bigoplus_{d=1}^{\sigma\left(G / N, W^{*}\right)} \mathbb{F}\left[W^{*}\right]_{d}^{G / N}$. Then $\mathbb{F}\left[W^{*}\right]^{G / N}$ is a finite $T$-module, implying that $S^{G}$ is a finite $\pi(T)$-module, and thus $\mathbb{F}[V]^{G}$ is a finite $\pi(T)$-module. Since by construction $\pi(T)$ is generated by elements of degree at most $\sigma(N, V) \sigma\left(G / N, W^{*}\right)$, the desired inequality follows.

Lemma 5.5. Let $G$ be a finite group, $H$ a subgroup of $G$, and $V$ a $G$-module. Then

$$
\sigma(H, V) \leq \sigma(G, V) \leq[G: H] \sigma(H, V) .
$$


Proof. The first inequality is trivial. By Proposition 4.6 there are homogeneous elements $f_{1}, \ldots, f_{r} \in \mathbb{F}[V]^{H}$ of degree at most $\sigma(H, V)$ such that the common zero locus of $f_{1}, \ldots, f_{r}$ in $\overline{\mathbb{F}} \otimes V$ is $\{0\}$. The formula $0=\prod_{g}\left(f_{i}-f_{i}^{g}\right)$ where $g$ ranges over a set of right $H$-coset representatives in $G$ shows that $f_{i}^{[G: H]}$ is contained in the ideal of $\mathbb{F}[V]$ generated by $\mathbb{F}[V]_{+}^{G}$. Since $\operatorname{deg}\left(f_{i}^{[G: H]}\right) \leq$ $[G: H] \sigma(H, V)$, it follows that the common zero locus of $\bigoplus_{d=1}^{[G: H] \sigma(H, V)} \mathbb{F}[V]_{d}^{G}$ is contained in the common zero locus $\{0\}$ of $\left\{f_{i}^{[G: H]} \mid i=1, \ldots, r\right\}$. Consequently, again by Proposition $4.6, \mathbb{F}[V]^{G}$ is a finitely generated module over its subalgebra generated by the homogeneous components $\mathbb{F}[V]_{d}^{G}$ with $d \leq[G: H] \sigma(H, V)$.

Remark 5.6. (i) The statement and proof of Lemma 5.4 remain valid under the weaker assumption that $[G: N]$ is not divisible by $\operatorname{char}(\mathbb{F})$.

(ii) The statement and proof of Lemma 5.5 remain valid in the modular case $\operatorname{char}(\mathbb{F})|| G \mid$. When $H=\{1\}$ is the trivial subgroup, we obtain the inequality $\sigma(G, V) \leq|G|$.

Lemma 5.4 and Lemma 5.5 have the folowing immediate corollary:

Corollary 5.7. For any subquotient $K$ of $G$ we have

$$
\frac{\sigma(G)}{|G|} \leq \frac{\sigma(K)}{|K|}
$$

Remark 5.8. Lemma 5.4 and Lemma 5.5 are the analogues for $\sigma$ of the reduction lemmata for $\beta$ in [20] mentioned at the beginning of Section 4 . For a variant of these statements concerning separating invariants see Section 3.9.4 in [6], [15, and [17.

\section{$6 \quad \sigma(G)$ for some semidirect products}

We need some facts and terminology relating zero-sum sequences over $A=Z_{p}$, the group of prime order $p$, written additively. See for example [12] as a general reference to this topic. Recall that by a sequence over $A$ we mean a sequence $S=\left(s_{1}, \ldots, s_{d}\right)$ of elements $s_{i} \in A$ where the order of the elements is disregarded and repetition is allowed. We say that $S$ is a zero-sum sequence if $\sum_{i=1}^{d} s_{i}=$ $0 \in A$. Denote by $\operatorname{supp}(S)$ the set of elements of $A$ that occur in $S$.

Lemma 6.1. For any non-empty subset $S \subseteq Z_{p} \backslash\{0\}$ there exists a zero-sum sequence $T$ of length at most $p$ with $\operatorname{supp}(T)=S$.

Proof. Let $s_{1}, \ldots, s_{k}$ denote the elements of $S$. If $s_{1}+\cdots+s_{k}=0$, then the sequence $T:=\left(s_{1}, \ldots, s_{k}\right)$ satisfies the requirements. Otherwise for $i=1, \ldots k$ denote $n_{i}$ the unique element in $\{1, \ldots, p-1\}$ with $n_{i} s_{i}=-\left(s_{1}+\cdots+s_{k}\right) \in Z_{p}$. The $n_{i}$ are distinct, hence the smallest among them, say $n_{1} \leq p-k$. Then the sequence $T:=\left(s_{1}, \ldots, s_{1}, s_{2}, \ldots, s_{k}\right)$ (where the multiplicity of $s_{1}$ is $\left.n_{1}+1\right)$ satisfies the requirements. 
Proposition 6.2. Let $G=Z_{p} \rtimes Z_{d}$ be a semidirect product of cyclic groups, where $p$ is a prime, $d$ is a divisor of $p-1$, and $Z_{d}$ acts faithfully (via conjugation) on $Z_{p}$. Then we have $\sigma(G)=p$.

Proof. We know that $\sigma(G) \geq \sigma\left(Z_{p}\right)=p$ by Lemma 5.5 and Corollary 5.3 By Lemma 5.1 it is enough to prove that $\sigma(G, U) \leq p$, where $U$ is an irreducible $G$ module. Since $\sigma(G)$ is not sensitive for extending the base field, we may assume that $\mathbb{F}$ is algebraically closed. Denote by $A$ the maximal normal subgroup $Z_{p}$ of $G$. Then $G$ has only two types of irreducible representations: if $U$ is 1-dimensional with $A$ in its kernel, then $\sigma(G, U) \leq|G / A|=\left|Z_{d}\right| \leq p-1$. Otherwise $U$ is induced from a non-trivial 1-dimensional $A$-module. In this case we may choose variables $x_{1}, \ldots, x_{d}$ in $\mathbb{F}[U]$ such that the $x_{i}$ are $A$-eigenvectors permuted up to scalar multiples by $G$, and denoting by $\theta_{i} \in \hat{A}:=\operatorname{hom}_{\mathbb{Z}}\left(A, \mathbb{F}^{\times}\right)$ the corresponding character of $A$ (i.e. $x_{i}^{a}=\theta_{i}(a) x$ for all $a \in A$ ), the set $O:=\left\{\theta_{1}, \ldots, \theta_{d}\right\}$ is a $G / A$-orbit in $\hat{A}$ (on which $G$ acts in the standard way). For a monomial $m=x_{1}^{\alpha_{1}} \ldots x_{d}^{\alpha_{d}} \in \mathbb{F}[U]$ denote by $\Phi(m)$ the sequence over $\hat{A}$ containing $\theta_{i}$ with multiplicity $\alpha_{i}$ for $i=1, \ldots, d$, and no other elements. Obviously $m$ is $A$-invariant if and only if $\Phi(m)$ is a zero-sum sequence.

For every $k \leq|O|$ we choose representatives $S_{k, 1}, \ldots, S_{k, r_{k}}$ from each $G / A$ orbit of the $k$-element subsets of $O$. By Lemma 6.1 we can assign to each of them an $A$-invariant monomial $m_{S_{k, i}} \in \mathbb{F}[U]$ with support $\operatorname{supp}\left(\Phi\left(m_{S_{k, i}}\right)\right)=S_{k, i}$ and degree at most $p$. Now consider the polynomials:

$$
f_{k}=\sum_{i=1}^{r_{k}} \sum_{g \in G / A} m_{S_{k, i}}^{g} \quad \text { for } k=1, \ldots,|O|
$$

They are all $G$-invariants, moreover, it is easily checked that their common zero locus is $\{0\}$. Indeed, if the vector $u=\left(u_{1}, \ldots, u_{|O|}\right) \in \overline{\mathbb{F}} \otimes U \cong \overline{\mathbb{F}}^{d}$ belongs to this common zero locus, and if the set $S=\left\{i: u_{i} \neq 0\right\}$ has cardinality $k>0$ then $0=f_{k}(u)=c \cdot m_{S}(u)$ for some divisor $c$ of $d$. It follows that $m_{S}(u)=0$, implying that $u_{j}=0$ for an index $j \in S$, which is a contradiction. Consequently $\mathbb{F}[U]^{G}$ is finitely generated over $\mathbb{F}\left[f_{1}, \ldots, f_{|O|}\right]$ by Proposition 4.6, hence $\sigma(G, U) \leq \max _{k} \operatorname{deg}\left(f_{k}\right) \leq p$.

Proposition 6.3. Let $G=A \rtimes Z_{2}$ be a semidirect product where $A$ is a nontrivial abelian group on which $Z_{2}$ acts by inversion. Then $\sigma(G)=\exp (A)$.

Proof. By Lemma 5.1 we know that $\sigma(G)=\max \sigma(G, U)$ where $U$ is an irreducible $G$-module. As above, we may assume that $\mathbb{F}$ is algebraically closed. If $U$ is 1-dimensional, then $\sigma(G, U) \leq \sigma\left(G / G^{\prime}\right)$, where $G^{\prime}$ is the commutator subgroup of $G$. It is easy to see that $G / G^{\prime}$ is an elementary abelian 2-group, whence $\sigma\left(G / G^{\prime}\right)=\exp \left(G / G^{\prime}\right)=2 \leq \exp (A)$. If the irreducible $G$-module $U$ is not 1-dimensional, then $\mathbb{F}[U]_{1}=U^{*}=\langle x, y\rangle$ where $x^{a}=\theta(a) x$ for any $a \in A$ and some character $\theta \in \hat{A}$, and $y^{a}=\theta(a)^{-1} y$; moreover $x$ and $y$ are exchanged by the generator $b$ of $Z_{2}$. Let $e$ denote the order of $\theta$ in $\hat{A}$; evidently $e \leq \exp A$. Now it is easily seen that $\mathbb{F}[U]^{G} \supseteq \mathbb{F}\left[x^{e}+y^{e}, x y\right]$, whence $\sigma(G, U) \leq \max \{e, 2\}$. 
Thus we proved the inequality $\sigma(G) \leq \exp (A)$. For the reverse inequality note that $\sigma(G) \geq \sigma(A)$ by the first inequality in Lemma 5.5.

\section{An improved general bound on $\sigma(G)$}

In this section we give an improvement for non-cyclic $G$ of the general inequality $\sigma(G) \leq|G|$.

Theorem 7.1. If $G$ is non-cyclic and $q$ is the smallest prime divisor of $|G|$, then

$$
\sigma(G) \leq \frac{|G|}{q}
$$

Proof. If $G$ has a subquotient isomorphic to $Z_{p} \times Z_{p}$ for some prime $p$ then by Corollary 5.7 and Corollary 5.3 we get that:

$$
\frac{\sigma(G)}{|G|} \leq \frac{\sigma\left(Z_{p} \times Z_{p}\right)}{p^{2}}=\frac{1}{p}
$$

and we are done. Note that for a non-cyclic $p$-group $P$, the factor group $P / \Phi(P)$ (where $\Phi(P)$ is the Frattini subgroup of $P$ ) contains a subgroup isomorphic to $Z_{p} \times Z_{p}$. So it remains to deal with the case when all Sylow subgroups of $G$ are cyclic. Then by a well known theorem of Burnside $G$ is the semidirect product of cyclic groups, and necessarily contains as a subquotient a non-abelian semidirect product $Z_{p} \rtimes Z_{q}$, where $p, q$ are primes, $q$ dividing $p-1$ (see for example [2] for references and details). Therefore by Corollary [5.7 and Proposition 6.2 we conclude $\sigma(G) /|G| \leq \sigma\left(Z_{p} \rtimes Z_{q}\right) / p q=1 / q$.

Remark 7.2. Theorem 7.1 is sharp for example for the abelian group $Z_{n q} \times Z_{q}$ or for a non-abelian semi-direct product $Z_{n} \rtimes Z_{q}$.

Theorem 7.1 is a variant for $\sigma(G)$ of the main combined result of [2] and [3] concerning $\beta(G)$. The present result for $\sigma(G)$ is easier, but the conclusion is stronger. We finish by stating a corresponding conjectured statement for the Noether number:

Conjecture 7.3. Let $\mathcal{C}_{q}$ denote the set of isomorphism classes of non-cyclic finite groups of order not divisible by char $(\mathbb{F})$ and with smallest prime divisor $q$. Then

$$
\limsup _{G \in \mathcal{C}_{q}} \frac{\beta(G)}{|G|}=\frac{1}{q}
$$

The case $q=2$ holds by 2 and 3 . For $q>2$ the conjecture is open.

\section{Acknowledgement}

We thank the referees for helpful comments on the manuscript. 


\section{References}

[1] D. J. Benson, Polynomial Invariants of Finite Groups, Cambridge University Press, 1993.

[2] K. Cziszter and M. Domokos, Groups with large Noether bound, arXiv:1105.0679v4 .

[3] K. Cziszter and M. Domokos, Noether's bound for the groups with a cyclic subgroup of index two, arXiv:1205.3011v1.

[4] Ch. Delorme, O. Ordaz, and D. Quiroz, Some remarks on Davenport constant, Discrete Math. 237 (2001), 119-128.

[5] H. Derksen, Polynomial bounds for rings of invariants, Proc. Amer. Math. Soc. 129 (2001), 955-963.

[6] H. Derksen and G. Kemper, Computational Invariant Theory, SpringerVerlag, Berlin, 2002.

[7] M. Domokos and P. Hegedüs, Noether's bound for polynomial invariants of finite groups, Arch. Math. 74 (2000), 161-167.

[8] P. Fleischmann, The Noether bound in invariant theory of finite groups, Adv. Math. 156 (2000), 23-32.

[9] P. Fleischmann, On invariant theory of finite groups, in "Invariant Theory in All Characteristics", (Ed.: H. E. A. Eddy Campbell and D. L. Wehlau), CRM Proceedings and Lecture Notes 35, Amer. Math. Soc., Providence, Rhode Island, pp. 43-69, 2004.

[10] J. Fogarty, On Noether's bound for polynomial invariants of a finite group, Electron. Res. Announc. Amer. Math. Soc. 7 (2001), 5-7.

[11] M. Freeze and W. A. Schmid, Remarks on a generalization of the Davenport constant, Discrete Mathematics 310 (2010) 3373-3389.

[12] W. Gao and A. Geroldinger, Zero-sum problems in finite abelian groups: A survey, Expo. Math. 24 (2006), 337-369.

[13] A. Geroldinger and F. Halter-Koch, Non-unique factorizations. Algebraic, combinatorial and analytic theory, Monographs and Textbooks in Pure and Applied Mathematics, Chapman \& Hall/CRC, 2006.

[14] F. Halter-Koch, A generalization of Davenport's constant and its arithmetical applications, Colloquium Mathematicum 63 (1992), 203-210.

[15] G. Kemper, Separating invariants, J. Symbolic Computation 44 (2009), 1212-1222. 
[16] F. Knop, On Noether's and Weyl's bound in positive characteristic, in "Invariant Theory in All Characteristics", (Ed.: H. E. A. Eddy Campbell and D. L. Wehlau), CRM Proceedings and Lecture Notes 35, Amer. Math. Soc., Providence, Rhode Island, pp. 175-188, 2004.

[17] M. Kohls and H. Kraft, Degree bounds for separating invariants, Math. Res. Lett. 17 (2010), 1171-1182.

[18] E. Noether, Der Endlichkeitssatz der Invarianten endlicher Gruppen, Math. Ann. 77 (1916), 89-92.

[19] V. Popov, The constructive theory of invariants, Math. USSR Izvest. 10 (1982), 359-376.

[20] B. J. Schmid, Finite groups and invariant theory, "Topics in Invariant Theory", Lect. Notes in Math. 1478 (1991), 35-66.

[21] M. Sezer, Sharpening the generalized Noether bound in the invariant theory of finite groups, J. Algebra 254 (2002), 252-263.

[22] H. Weyl, The Classical Groups, Princeton University Press, Princeton, 1939. 